RESEARCH PAPER RP1429

Part of Journal of Research of the National Bureau of Standards, Volume 27, November 1941

\title{
SUGAR ACETATES, ACETYLGLYCOSYL HALIDES, AND ORTHOACETATES IN RELATION TO THE WALDEN INVERSION ${ }^{12}$
}

\author{
By Harriet L. Frush and Horace S. Isbell
}

\section{ABSTRACT}

The tendency of the acetyl groups in the sugar acetates and glycosyl halides to form intramolecular condensation products depends upon the stereomeric arrangement of the groups within the molecule. In this paper the stereomeric factors which control the formation of orthoesters are discussed in light of the oppositeface concept for the Walden inversion, and it is shown that cyclic orthoacetates are formed when an acetyl group can approach the face of a neighboring carbon opposite a replaceable halogen atom. This concept was tested by application of the Koenigs-Knorr reaction to pentaacetyl- $\alpha-d-\alpha$-guloheptopyranosyl bromide and to heptaacetyl- $\alpha$-neolactosyl chloride. Both of these compounds gave, as anticipated, the corresponding methyl orthoacetates.

\section{CONTENTS}

roduction

II. Walden inversion

III. Replacement reactions of the acetylglycosyl halides

IV. Experimental details _._.

1. Hexaacetyl- $\alpha-d-\alpha$-guloheptopyranose

2. Pentaacetyl- $\alpha-d$ - $\alpha$-guloheptopyranosyl bromide $\ldots \ldots 42$

3. Tetraacetyl- $d$ - $\alpha$-guloheptose methyl 1,2-orthoacetate . . . . . _. 423

4. Hexaacetylneolactose methyl 1,2-orthoacetate....... 425

5. Methyl heptaacetyl- $\beta$-neolactopyranoside
V. Summary
VI. References

\section{INTRODUCTION}

The sugar acetates and their derivatives are among the most important sugar compounds from the standpoint of their general usefulness for analytical purposes, in synthesis, and in the elucidation of structure. Methods for the preparation of the fully acetylated derivatives were discovered early in the history of sugar chemistry, and several of the sugar acetates were obtained in crystalline form. In some cases two crystalline acetates were prepared from the same sugar,

1 The material in this paper was abstracted from a thesis submitted by Harriet L. Frush, of this Bureau, in May 1941 to the Faculty of the Graduate School of the University of Maryland, represented by Nathan L. Drake, in partial fulfilment of the requirements for the degree of Doctor of Philosophy.

2 This paper was presented before the division of Sugar Chemistry and Technology of the American Chemical Society at Atlantic City, N. J., September 1941. 
and it became apparent that these were alpha and beta modifications analogous to the methyl glycosides. Today a large number of the crystalline sugar acetates are known in both alpha and beta modifications, and are widely used in the characterization of the sugars and their derivatives. Because the acetyl linkage can be removed readily, and yet is comparatively stable, acetylation has frequently been employed to protect part of the hydroxyls in the sugar molecule in the course of reactions involving the remaining groups. Thus the acetylated sugars have proved to be valuable starting materials in the synthesis of other derivatives.

Even more useful than the sugar acetates in synthetic processes are their halogen derivatives, the acetylglycosyl halides, prepared by reaction of the hydrogen halide upon the acetate, and having the halogen attached to the glycosidic carbon. Since this halogen is particularly reactive in comparison with the acetyl groups, the acetylglycosyl halides may be used in a wide variety of reactions involving replacement on the glycosidic carbon. Thus an acetylglycosyl halide may be condensed with alcoholic hydroxyl groups in the presence of a basic condensing agent (for example, silver oxide or carbonate, or quinoline) to form glycosides, substances which are widely distributed in plant and animal life. Consequently, the acetylglycosyl halides of the sugars have an essential part in the synthesis of compounds which are important in biochemical research and have practical applications in the fields of pharmacology and medicine. Numerous syntheses of natural glycosides have already been carried out, frequently through the reaction of the nonsaccharide constituent with an acetylglycosyl halide.

A special group of glycosides, the disaccharides, may be prepared by condensation of acetylglycosyl halides with certain sugar derivatives. By treating tetraacetyl- $\alpha-d$-glucosyl bromide with tetraacetyl$d$-glucose in the presence of silver carbonate, Fischer synthesized isotrehalose ( $\beta, \beta$-trehalose) [1], ${ }^{3}$ and by similar methods other workers, especially Helferich, Freudenberg, and Evans, have prepared many compound sugars. In these condensations the alpha acetylglycosyl halide is usually combined with a sugar having one free hydroxyl group, and the resulting disaccharide is formed with a Walden inversion on carbon 1 , so that these syntheses generally lead to disaccharides with a beta type of union. One might anticipate that the alpha type of union could be obtained by the use of the beta acetylglycosyl halide, but the preparations have not generally been successful. A few beta acetylglycosyl chlorides have been obtained by treatment of the corresponding alpha acetylglycosyl bromide with an especially prepared silver chloride [2], but many of these beta compounds are unstable and in condensation reactions give complex mixtures from which crystalline products are not readily separable.

Since replacement occurs with inversion at carbon 1, the difference in behavior of the alpha and beta acetylglycosyl halides is, no doubt, related to the mechanism of the Walden inversion. A brief review of the Walden inversion, especially in relation to the opposite-face mechanism, will afford a basis for the discussion of the replacement reactions of the acetylglycosyl halides.

8 Figures in brackets indicate the literature references at the end of this paper. 


\section{WALDEN INVERSION}

In 1896 Walden reported an inversion of optical rotation in the conversion of malic to chlorosuccinic acid by reaction with phosphorus pentachloride [3].

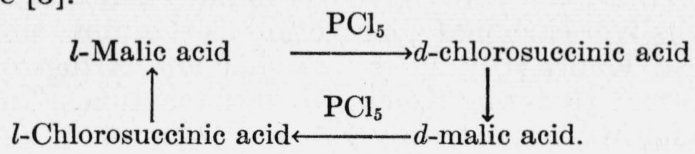

Since this brief report the phenomenon, which has come to be known as the Walden inversion, has been extensively studied with numerous reactants and optically active substances $[4,5]$. Perhaps the most satisfactory explanation for the change in configuration was suggested by Werner in 1911 [6] and is commonly known as the opposite-face mechanism for the Walden inversion. Werner pointed out that in the tetrahedron formed by the groups $A, B, D$, and $X$ attached to a carbon, there are four positions at which an entering group may become attached, with the displacement of group $X$, namely the four faces $A B X, B D X, D A X$, or $A B D$. It will be observed in figure 1 that substitution for $X$ at one of the first three of these faces will cause no change in configuration, but when the entering group, $Y$, approaches the face opposite $X(A B D)$, with simultaneous withdrawal of $X$, a shift to the enantiomorphic configuration must occur. Garner [7] clarified the Werner concept by means of an ingenious mechanical model showing this shift in valences, which is comparable to the turning inside out of an umbrella. ${ }^{4}$
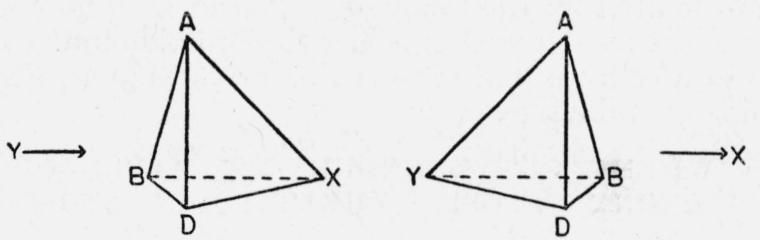

Figure 1.-Opposite-face mechanism for the Walden inversion.

The sugars and their derivatives are uniquely adapted to the study of the inversion mechanism, since, with the carbon atoms bound in a ring, rotation about the carbon-carbon bonds is largely restricted, and the spheres of influence of the various groups within the molecule are oriented in relation to one another. Recently, Isbell [9] pointed out that in certain replacement reactions intramolecular condensations take place when reactive groups within the sugar molecule can approach the face of the carbon opposite its replaceable group (halide, tosyl, etc.) and that the opposite-face concept appears to provide a satisfactory explanation for the formation or lack of formation of intramolecular condensation products, among which are the anhydrosugars and the orthoesters. Inasmuch as some of these intramolecular condensation products are formed when the acetylglycosyl halides are used for the preparation of glycosides, it seemed desirable to investigate more fully the replacement reactions of the acetylglycosyl halides in relation to the opposite-face concept.

4 A comprehensive discussion of the Walden inversion and the work which has led to the acceptance of the opposite-face mechanism may be found in chapters V and VI of reference [8]. 


\section{REPLACEMENT REACTIONS OF THE ACETYLGLYCOSYL HALIDES}

The replacements studied in this investigation have involved the reaction between certain acetylglycosyl halides and methyl alcohol in the presence of silver carbonate, a reaction used almost simultaneously by Koenigs and Knorr [10] and by Fischer and Armstrong [11], and commonly referred to as the Koenigs-Knorr reaction. In some cases, the first of which was reported by Fischer, Bergmann, and Rabe [12], this reaction leads to the formation of substances which do not have the properties of normal glycosides. As shown simultaneously by Freudenberg and Braun [13], and by Haworth and coworkers [14, $15]$, one of the acetyl groups is joined to the glycosidic carbon and to the adjacent carbon in a cyclic orthoacetic ester structure. According to Isbell [9], the orthoacetate seems to be formed by an intramolecular reaction in which an acetyl group approaches the face of carbon 1 opposite the departing halogen, and combines with inversion. Presumably, as the bromine is removed by combination with the silver carbonate or other suitable reagent, the carbonyl oxygen is attracted to the opposite face of carbon 1, leaving the acetyl carbon deficient in electrons. The deficiency is satisfied by the attraction of a negative group, in this case methoxyl, from the environment. Electronically the reaction may be considered to consist in a flow of electrons from the hydrogen of the methyl alcohol to the methoxyl, from the methoxyl to the acetyl carbon, to the oxygen, to the glycosidic carbon, and to the bromine. This results in the formation of a hydrogen ion, a bromide ion, and the methyl orthoacetate. The process is represented by the following equations. The reactions are driven to the right by removal of either the bromide ion or the hydrogen ion, the former by use of silver oxide or carbonate, the latter by use of pyridine or quinoline.

\section{REACTIONS WHEN ACETYL GROUP CAN APPROACH FACE OF CARBON 1 OPPOSITE THE HALOGEN ATOM}

Orthoester reaction

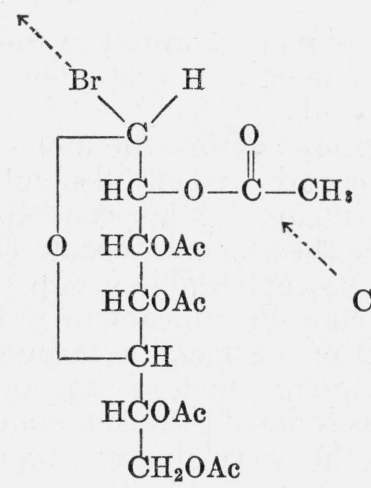

Pentaacetyl- $\alpha$ - $d$ - $\alpha$-guloheptosyl bromide
$\mathrm{Br}^{-}$

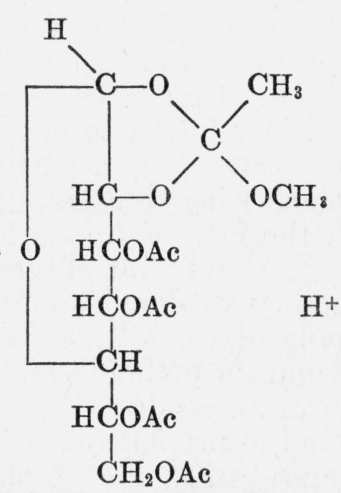

Tetraacetyl- $d$ - $\alpha$-guloheptose methyl orthoacetate 


\section{Competitive glycosidic reaction}

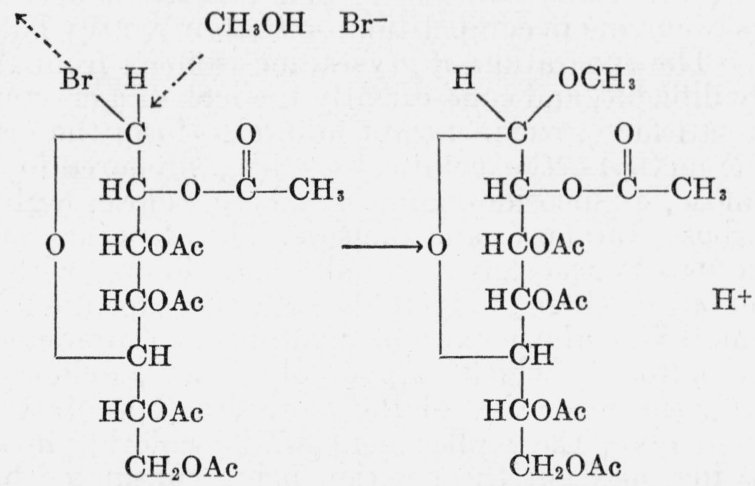

Pentaacetyl- $\alpha$ - $d$ - $\alpha$-guloheptosyl bromide Methyl pentaacetyl- $\beta-d-\alpha$-gulo-
heptoside

\section{REACTION WHEN ACETYL GROUP CANNOT APPROACH FACE OF} CARBON 1 OPPOSITE THE HALOGEN ATOM

Glycosidic reaction

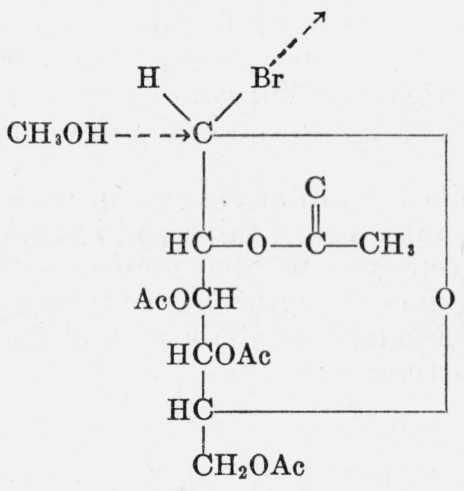

Tetraacetyl- $\alpha$ - $d$-glucosyl bromide

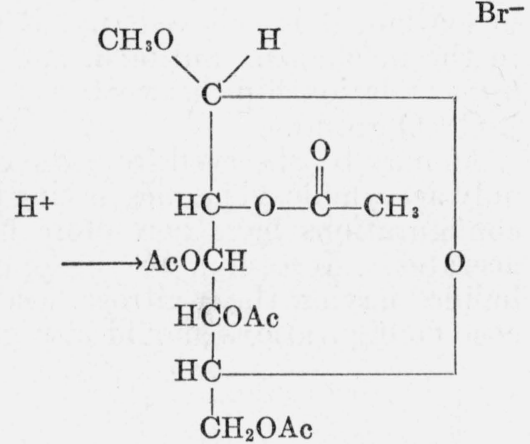

Methyl tetraacetyl- $\beta$ - $d$-glucoside

The orthoacetate is formed only when the carbonyl oxygen of an acetyl group can approach the face of the carbon opposite the halogen. This is possible when the acetyl group and halogen atom lie on opposite sides of the plane of the sugar ring. As shown in the equations, when the halogen and the acetyl group of carbon 2 of an aldose are on the same side of the ring, the carbonyl oxygen of the acetyl group is unable to approach carbon 1 at the face opposite the departing halogen, and therefore it cannot form the orthoacetate. Under these circumstances the halogen is replaced with inversion by a negative group from the environment, methoxyl in this case. This is the ordinary KoenigsKnorr reaction, which leads to the formation of normal glycosides. However, when the halogen and the adjacent acetyl group are on opposite sides of the ring, either the acetyl group of the adjacent carbon or the methoxyl group from the environment is capable of approaching the face of carbon 1 opposite the departing halogen, and 
combining with inversion. One of these reactions gives the normal glycoside, the other the orthoester, and the two reactions may be conceived as occurring in competition, so that ordinarily both products are formed. The separation of crystalline isomers from the mixture is frequently difficult, and consequently the lack of a crystalline derivative is not satisfactory evidence of failure to form the orthoester.

Crystalline methyl orthoacetates have been prepared for rhamnose, lyxose, mannose, 4-glucosido-mannose, ribose, talose, $\alpha$-glucoheptose, fructose, sorbose, turanose, and maltose. The structure and configuration of the parent acetylglycosyl halides are known with reasonable certainty for all of these compounds with the exception of maltose. Since the methyl orthoacetate of maltose was prepared from an "acetochloromaltose," which supposedly has a chloro-orthoester structure [16], the formation of the methyl orthoacetate of maltose presumably involves the replacement of the chlorine in a structure already existing, and not the reaction being considered here. Inasmuch as carbon 1 of the ketoses is free to rotate about the carboncarbon axis, the acetyl group of carbon 1 can always be brought into a favorable position for the orthoester reaction, and all ketoses are therefore capable of orthoester formation. Aside from the derivatives of maltose and the ketoses, the methyl orthoacetates so far prepared have been formed from acetylglycosyl halides in which the acetyl groups of both carbons 2 and 3 are trans to the halogen. Although the configuration of carbon 3 may to some extent influence orthoester formation, it is believed that it plays only a limited part, according to the mechanism outlined, and that the essential configuration is a trans relationship between the halogen atom and a neighboring $>\mathbf{C}=\mathbf{O}$ group.

As may be observed from the compilation of data shown in table 1, only acetylaldosyl halides having the $\alpha$-mannose, $\alpha$-talose, and $\beta$-gulose configurations have heretofore been reported to give methyl orthoacetates. According to the opposite-face hypothesis, acetylaldosyl halides having the $\alpha$-altrose, $\alpha$-idose, $\beta$-galactose, $\beta$-allose, and $\beta$-glucose configurations should also give orthoesters. 
TABLE 1-Aldose sugars which have yielded methyl orthoacetates

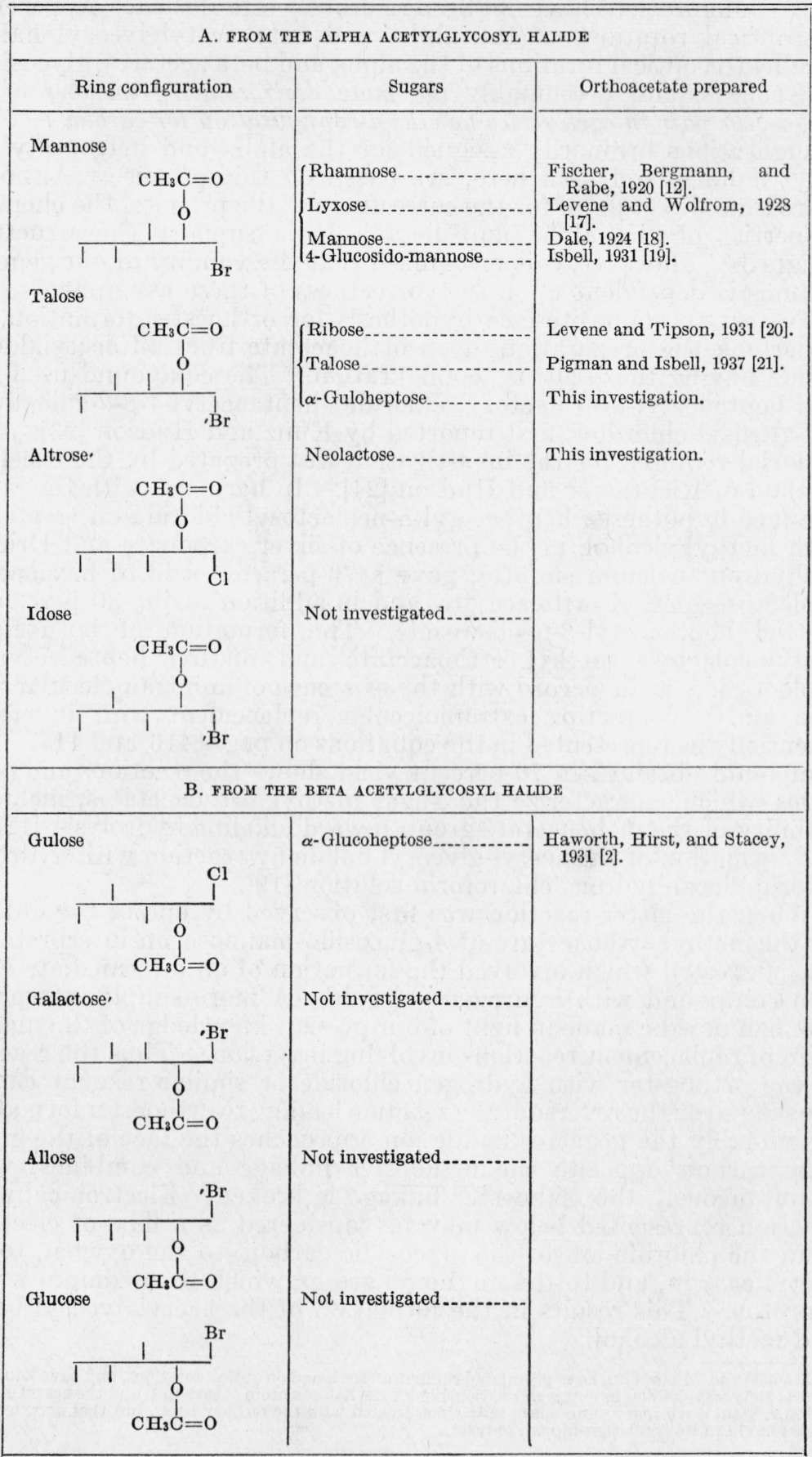


In the foregoing discussion the configurations of the alpha and beta acetylglycosyl halides have been tacitly assumed, but unfortunately these configurations have not been rigorously established. Apparently the optical rotations of the alpha and beta acetylglycosyl halides parallel the optical rotations of the alpha and beta acetates, glycosides, and sugars, and presumably the more dextrorotatory member of the alpha-beta pair in each series has the d-configuration for carbon 1. The configurations ordinarily assigned for the alpha and beta acetylglycosyl halides, and used here, are based on this premise. Although there is no direct proof for the correctness of the premise, the chemical properties of the alpha and beta isomers support the structures assigned. ${ }^{5}$ However, it is recognized that the validity of our generalizations is dependent upon the correctness of these assignments.

To test the opposite-face hypothesis for orthoester formation, we undertook the preparation of an orthoacetate from an acetylaldosyl halide having the $\alpha$-altrose configuration. The compound used was was heptaacetyl- $\alpha$-neolactosyl chloride (heptaacetyl-4- $\beta$ - $d$-galactosyl$\alpha$ - $d$-altrosyl chloride), first reported by Kunz and Hudson [23]. The material required for the investigation was prepared by the excellent method of Richtmyer and Hudson [24]. In harmony with the opposite-face hypothesis, heptaacetyl- $\alpha$-neolactosyl chloride on treatment with methyl alcohol, in the presence of silver carbonate and Drierite (anhydrous calcium sulfate), gave a 70-percent yield of hexaacetylneolactose methyl orthoacetate, and in addition about 30 percent of methyl heptaacetyl- $\beta$-neolactoside. The formation of both hexaacetylneolactose methyl orthoacetate and methyl heptaacetyl $\beta$ neolactoside is in accord with the existence of anintramolecular reaction and a competing extramolecular replacement with inversion, essentially as represented in the equations on pages 416 and 417 . The compound obtained in 70-percent yield shows the reactions and properties which characterize the sugar methyl orthoacetates, including stability of the orthoacetate group toward alkaline hydrolysis [12, 18] and formation of the acetyl-glycosyl halide by reaction with hydrogen chloride in anhydrous chloroform solution [19].

When the latter reaction was first observed by one of the authors for the methyl orthoacetate of 4-glucosido-mannose, an interpretation was suggested which involved the formation of an intermediate addition compound with hydrogen chloride. A more simple interpretation can now be made in light of our present knowledge of the mechanism of replacement reactions involving inversion. Thus the reaction of the orthoester with hydrogen chloride or similar reagent can be considered as the reverse of the reaction leading to orthoester formation. Presumably the negative halide ion approaches the face of the glycosidic carbon opposite the orthoester linkage and combines, while simultaneously the orthoester linkage is broken. Electronically the reaction represented below may be considered as a flow of electrons from the chloride ion to the glycosidic carbon, to the oxygen, to the acetyl carbon, and to the methoxyl group, which then combines with a proton. This results in the formation of the acetylglycosyl halide and methyl alcohol.

\footnotetext{
- Micheel and Micheel [22] have postulated configurations based on optical rotations, and have found that the acetylglycosyl halides having a cis relationship for the halogen atom of carbon 1, and the acetyl group of carbon 2, yield quaternary ammonium salts when treated with the tertiary bases, but that acetylglycosyl halides having a trans relationship fail to react.
} 


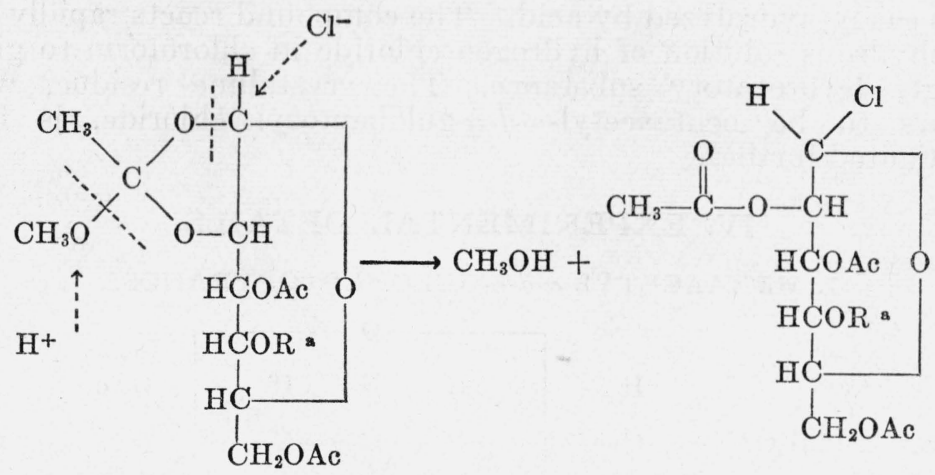

Hexaacetylneolactose methyl 1,2-

Heptaacetyl- $\alpha$-neolactosyl orthoacetate

chloride

- $\mathrm{R}$ is the tetraacetyl- $\boldsymbol{\beta}-\boldsymbol{d}$-galactosyl group.

To obtain further information as to the general applicability of the opposite-face hypothesis in relation to orthoester formation, we undertook the preparation and study of a number of acetylglycosyl halides in the heptose series. This work, which is still in progress, has led to the preparation of an orthoester of $d$ - $\alpha$-guloheptose.

The formation of the orthoester of $d-\alpha$-guloheptose required the successive preparation of hexaacetyl- $\alpha-d-\alpha$-guloheptose and pentaacetyl$\alpha$ - $d$ - $\alpha$-guloheptosyl bromide, and the application of the KoenigsKnorr reaction to the latter substance. Hexaacetyl- $\alpha-d$ - $\alpha$-guloheptose was obtained by low-temperature acetylation, with acetic anhydride and pyridine, of crystalline $\alpha-d-\alpha$-guloheptose. The manner of preparation of the new acetate supports its classification as the modification analogous to that of the sugar; that is, as hexaacetyl- $\alpha-d-\alpha-$ guloheptose. Pentaacetyl- $\alpha-d-\alpha$-guloheptosyl bromide was obtained by treating hexaacetyl- $\alpha-d$ - $\alpha$-guloheptose with hydrogen bromide at $0^{\circ} \mathrm{C}$. The product crystallizes readily and, when pure, is relatively stable. The reaction of the new bromide with methyl alcohol in the presence of silver carbonate and Drierite was studied by analysis of the solution after reaction was complete, and by isolation and analysis of the reaction product.

The orthoacetyl group is readily hydrolyzed by acid but is stable to alkali, whereas the ordinary acetyl group is hydrolyzed by either reagent. Consequently the analysis of the solution obtained from the Koenigs-Knorr reaction was made by acetyl determinations in acid and in alkaline solution. Upon the basis of the difference in the results of the acid and alkaline hydrolyses, the conversion of pentaacetyl- $\alpha-d$ - $\alpha$-guloheptosyl bromide to the acetylated orthoester was calculated to be nearly quantitative.

Upon evaporation of the solution, a crystalline product was obtained in nearly quantitative yield, and the product gives the reactions characteristic of the orthoester derivatives. As already noted, one acetyl group (the orthoacetyl) is resistant to alkaline hydrolysis 
but is easily hydrolyzed by acid. The compound reacts rapidly with an anhydrous solution of hydrogen chloride in chloroform to give a strongly levorotatory substance. The crystalline residue, which appears to be pentaacetyl- $\alpha-d$ - $\alpha$-guloheptosyl chloride, is being investigated further.

\section{EXPERIMENTAL DETAILS}

1. HEXAACETYL- $\alpha-d-\alpha$-GULOHEPTOPYRANOSE

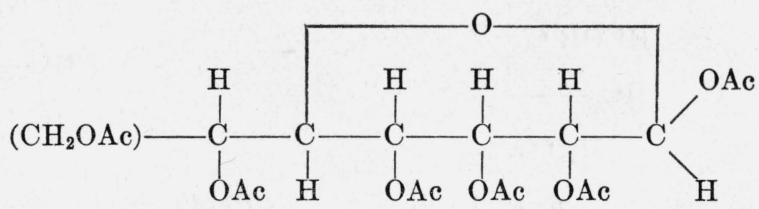

Five grams of finely powdered $\alpha-d$ - $\alpha$-guloheptose ${ }^{6}$ was acetylated by the use of $28 \mathrm{ml}$ of acetic anhydride and $35 \mathrm{ml}$ of pyridine. The acetylation mixture was stirred in an ice bath for 2 days, then poured, with stirring, into ice water. After a short time the acetate separated as a gum, the water was decanted, and a fresh quantity of ice water was added, from which in the course of about an hour, with continuous stirring, a crystalline substance separated. The acetate, largely hexaacetyl- $\alpha-d-\alpha$-guloheptopyranose, weighed $7.5 \mathrm{~g}$ and melted at $115^{\circ} \mathrm{C}$, approximately. A total of $13.2 \mathrm{~g}$ of crystalline material, obtained from two acetylations, was recrystallized several times from ethyl alcohol, in which the compound is quite soluble. Hexaacetyl- $\alpha-d-\alpha$-guloheptopyranose crystallizes from ethyl alcohol in chunky rectangular prisms (fig. $2, A$ ) which melt at $126^{\circ} \mathrm{C}$ and give $[\alpha]_{D}^{20}=-62.8^{\circ}$. Analysis: Calculated for $\mathrm{C}_{19} \mathrm{H}_{26} \mathrm{O}_{13}: \mathrm{C}, 49.35 ; \mathrm{H}$, 5.67. Found: C, $49.47 ; \mathrm{H}, 5.64$.

\section{PENTAACETYL- $\alpha-d-\alpha$-GULOHEPTOPYRANOSYL BROMIDE}

The new hexaacetyl- $\alpha-d-\alpha$-guloheptopyranose $(6.8 \mathrm{~g})$ was treated at $0^{\circ} \mathrm{C}$ with $35 \mathrm{ml}$ of a saturated solution of hydrogen bromide in acetic acid and with $3.5 \mathrm{ml}$ of acetic anhydride. After the acetate had dissolved, the solution was kept in ice for 3 hours, and was then poured, with stirring, into a cold mixture of water and benzene. The benzene solution was separated and washed six times with ice water, dried with Drierite, and after a filtration, concentrated in vacuum. When crystals began to separate from the sirup during evaporation, ether was added and the crystalline product was collected on a filter. The first crop weighed $4.0 \mathrm{~g}$, and an additional $0.7 \mathrm{~g}$ was obtained by concentrating the mother liquor. The acetylglycosyl halide was recrystallized at once by dissolving it in benzene and adding ether. Pentaacetyl- $\alpha-d$ - $\alpha$-guloheptopyranosyl bromide crystallizes in slender

\footnotetext{
6 The aldonic acid from which this sugar was prepared was originally named by La Forge, $d$-6-guloheptonic acid. However, using the nomenclature for the heptoses suggested by Isbell [25], in which the alpha and beta represent not the order of discovery but the configuration of carbon 2, the acid is named $d$ - $\alpha$-guloheptonic acid, and the sugar $d$ - $\alpha$-guloheptose. The sugar used here was part of that originally prepared by Isbell [26].
} 
prisms (fig. $2, B$ ) which melt at $139^{\circ}$ to $140^{\circ} \mathrm{C}$ and give $[\alpha]_{D}^{20}=-124^{\circ}$. The new compound is fairly stable when pure, and has been kept at room temperature for several weeks in a shallow dish over sodium hydroxide. Analysis: Calculated for $\mathrm{C}_{17} \mathrm{H}_{23} \mathrm{O}_{11} \mathrm{Br}$ : C, 42.25; H, $4.80 ; \mathrm{Br}$. 16.54 . Found: C, $42.27 ; \mathrm{H}, 4.92 ; \mathrm{Br}, 16.47$.

Since $d$ - $\alpha$-guloheptose has the $l$-talose configuration, a comparison of the molecular rotations of corresponding derivatives of the two sugars can be used for the assignment of ring structure and configuration. The following comparison of the molecular rotations of the new derivatives of $d$ - $\alpha$-guloheptose with the molecular rotations of the corresponding derivatives of talose prepared by Pigman and Isbell [21] may be used for this purpose:

Pentaacetyl- $\alpha$ - $l$-talose ${ }^{7}$ $-27,400$

Hexaacetyl- $d$ - $\alpha$-guloheptose

Tetraacetyl- $\alpha$-l-talosyl bromide ${ }^{7}$

Pentaacetyl- $d$ - $\alpha$-guloheptosyl bromide

Triacetyl-l-talose methyl orthoacetate ${ }^{7}$

Tetraacetyl- $d$ - $\alpha$-guloheptose methyl orthoacetate $\ldots \ldots \ldots+1,400$

7 The molecular rotations of the $l$-talose derivatives have been obtained from those of the $d$-talose derivatives by reversing the signs.

The parallelism between the optical rotations of tetraacetyl- $d$-talosyl bromide and pentaacetyl- $d$ - $\alpha$-guloheptosyl bromide indicates that the two substances have like ring structures, and the same configuration for the glycosidic carbon. Unfortunately the structure of the tetraacetyl- $d$-talosyl bromide is not definitely established. However, it may be seen from the values given below that the "epimeric difference" [28] of the acetylglycosyl bromides of glucose and mannose and the epimeric difference of the acetylglycosyl bromides of galactose and talose are of the same order of magnitude. If the configurations ordinarily accepted for the acetylglycosyl bromides of glucose, mannose, and galactose are correct, tetraacetyl- $d$-talosyl bromide and pentaacetyl- $d$ - $\alpha$-guloheptosyl bromide have the alpha pyranose configuration, and the bromine atom of carbon 1 and the acetyl group of carbon 2 are trans.

Epimeric [M] difference

Tetraacetyl- $\alpha$ - $d$-glucopyranosyl bromide $+81,300$

Tetraacetyl- $\alpha-d$-mannopyranosyl bromide $\ldots$

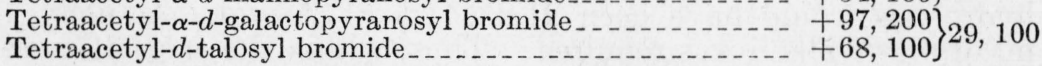

3. TETRAACETYL- $d-\alpha$-GULOHEPTOSE METHYL 1,2-ORTHOACETATE<smiles>COC(C1COC(C)(OC)O1)C(OC)C(C)(C)OC</smiles> 
Two grams of powdered Drierite ${ }^{8}$ and $4 \mathrm{~g}$ of freshly prepared silver carbonate were added to $50 \mathrm{ml}$ of methyl alcohol, and the mixture was mechanically stirred in an ice bath for 10 minutes. One gram of finely powdered pentaacetyl- $\alpha-d-\alpha$-guloheptopyranosyl bromide was added, and the reaction mixture was mechanically stirred at $0^{\circ} \mathrm{C}$ for 44 hours. The solution was then filtered, and portions of the filtrate $(10 \mathrm{ml})$ were pipetted into small glass-stoppered flasks for the analysis described below. When the solvent was evaporated from the remaining solution, the sirup crystallized almost completely upon the addition of a little ethyl alcohol with stirring. The crystalline material melted at approximately $100^{\circ} \mathrm{C}$ and gave no test for halogen.

In the analysis of the solution for orthoacetate, advantage was taken of the fact that $0.1 \mathrm{~N}$ acid at room temperature hydrolyzes both the ordinary acetyl and the orthoacetyl group, whereas $0.1 \mathrm{~N}$ alkali hydrolyzes the acetyl without disturbing the orthoacetyl group. The result of the acid hydrolysis corresponds to the sum of the methyl acetylglycosides and the orthoacetate, whereas the difference between the results obtained by acid and by alkaline hydrolysis is a measure of the amount of orthoacetate. Methyl pentaacetyl- $d$ - $\alpha$-guloheptoside gives 5 moles of acetic acid by either acid or alkaline hydrolysis, but tetraacetyl- $d-\alpha$-guloheptose methyl orthoacetate gives 5 moles of acetic acid by acid hydrolysis and only 4 moles by alkaline hydrolysis. For analysis, the samples which had been transferred to small flasks were treated in the following manner:

Alkaline hydrolysis.-Fifty milliliters of $0.1 \mathrm{~N}$ barium hydroxide was added to a 10-ml sample of the solution in a glass-stoppered bottle, and the mixture was allowed to stand at $20^{\circ} \mathrm{C}$ for 3 hours. A back titration made with $0.1 \mathrm{~N}$ sulfuric acid showed that acid equivalent to $17.52 \mathrm{ml}$ of $0.1 \mathrm{~N}$ barium hydroxide had been formed.

Acid hydrolysis. - Ten milliliters of $0.1 \mathrm{~N}$ sulfuric acid was added to a $10-\mathrm{ml}$ sample of the solution, and the mixture was allowed to stand at $20^{\circ} \mathrm{C}$ for 24 hours. Fifty milliliters of $0.1 \mathrm{~N}$ barium hydroxide was then added, and at the end of 3 hours the excess alkali was titrated with $0.1 N$ sulfuric acid. The results showed that during the successive treatment with acid and alkali, acid equivalent to $21.95 \mathrm{ml}^{9}$ of $0.1 \mathrm{~N}$ barium hydroxide had been formed. If the material had been exclusively the orthoacetate, the requirement by alkaline hydrolysis would have been $21.95 \times 4 / 5=17.56 \mathrm{ml}$. Actually 17.52 $\mathrm{ml}$ of $0.1 \mathrm{~N}$ alkali was required. Thus, within the error of measurement, the reaction product consists of the orthoacetate.

In a subsequent preparation of tetraacetyl- $d$ - $\alpha$-guloheptose methyl orthoacetate the analysis was omitted and the crystalline product, which separated upon evaporation of the methyl alcohol, was recrystallized several times from ethyl alcohol with the addition of petroleum ether. The compound, which crystallizes in chunky truncated prisms (fig. 2, C), is very soluble in ether and in alcohol but nearly insoluble in petroleum ether. When pure, tetraacetyl- $d-\alpha-$ guloheptose methyl 1,2-orthoacetate melts at $106^{\circ} \mathrm{C}$ and gives

\footnotetext{
${ }^{8}$ In the course of the reaction, water is formed and reacts further to give objectionable byproducts. This factor was not taken into account until relatively recently when Helferich and coworkers used a desiccant to absorb the water as it was formed. Helferich, Bohn, and Winkler [29] used calcium chloride; and Reynolds and Evans [30] introduced the use of powdered Drierite (calcium sulfate), which was used in all of the Koenigs-Knorr reactions conducted in the course of this investigation.

$?$ This value is somewhat higher than that $(20.69 \mathrm{ml})$ calculated from the bromide used and the original volume of the solution. The higher value may be attributed to the evaporation of alcohol during filtration of the solution. However, the original concentration is unimportant, since the actual concentration is determined experimentally by the acid hydrolysis.
} 
$[\alpha]_{D}^{20}=3.2^{\circ}$. Analysis: Calculated for $\mathrm{C}_{18} \mathrm{H}_{26} \mathrm{O}_{12}: \mathrm{C}, 49.77 ; \mathrm{H}, 6.03$. Found: C, 49.83; H, 6.09.

When treated with an anhydrous $0.1 N$ solution of hydrogen chloride in purified chloroform, the compound reacted rapidly in a manner characteristic of orthoesters [19]. Four minutes after dissolution the specific rotation was constant at $-38.1^{\circ}$, whereas the specific rotation of the orthoacetate in chloroform is $+3.2^{\circ}$. Upon evaporation, the solution gave a residue containing crystals, presumably pentaacetyl$\alpha$ - $d$ - $\alpha$-guloheptosyl chloride, which is being investigated further. Since the compound was prepared by the method previously used for the preparation of the orthoacetate of rhamnose, in which the orthoester group has been shown to be united to carbons 1 and 2 [31], it is assumed that the orthoester group in this compound is also attached to carbons 1 and 2 .

\section{HEXAACETXLNEOLACTOSE METHYL 1,2-ORTHOACETATE}

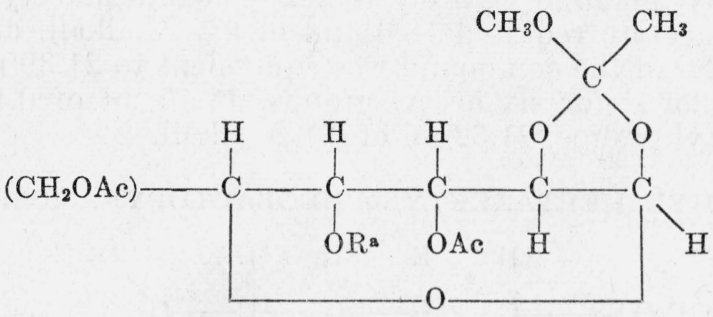

a $\mathrm{R}$ is the tetraacetyl- $\beta$ - $d$-galactosyl group.

Five grams of heptaacetyl- $\alpha$-neolactosyl chloride was used in the Koenigs-Knorr reaction under conditions identical with those described for pentaacetyl- $\alpha-d-\alpha$-guloheptosyl bromide, with the exception that the reaction time was increased from 44 hours to 72 hours. The methyl alcoholic solution, when evaporated in air to a sirup and stirred with a little ethyl alcohol, yielded slender prismatic crystals melting at $118^{\circ}$ to $121^{\circ} \mathrm{C}$ and having a specific rotation of approximately $+25^{\circ}$. Acid and alkaline hydrolyses of the reaction solution showed that only about 70 percent of the original sugar was present, and this was almost completely in the form of the orthoester. It was at first supposed that undissolved heptaacetyl- $\alpha$-neolactosyl chloride remained in the silver residues, but by extraction of these residues with chloroform, a halogen-free sirup was obtained from which short rectangular crystals separated upon the addition of methyl alcohol. This crude material melted at $168^{\circ}$ to $174^{\circ} \mathrm{C}$ and gave $[\alpha]_{D}^{20}=-13.7^{\circ}$. It thus appeared that application of the Koenigs-Knorr reaction to heptaacetyl- $\alpha$-neolactosyl chloride yielded, besides the crystalline orthoacetate, a second crystalline product, difficultly soluble in methyl alcohol and differing widely from the orthoacetate in optical rotation and melting point.

The hexaacetylneolactose methyl orthoacetate was recrystallized several times by dissolving it in absolute ethyl alcohol, concentrating the solution in vacuum to about one-third of its volume, and separating the crystals (slender prisms, fig. $2, D$ ). When thoroughly dried, the compound melts at $121^{\circ}$ to $122^{\circ} \mathrm{C}$, whereas after air-drying, it usually melts several degrees lower. For the pure substance 
$[\alpha]_{D}^{20}=+25.3^{\circ}$. Analysis: Calculated for $\mathrm{C}_{27} \mathrm{H}_{38} \mathrm{O}_{18}: \mathrm{C}, 49.84 ; \mathrm{H}, 5.89$. Found: C, $49.75 ; \mathrm{H}, 5.77$.

When the new compound was treated with an anhydrous $0.1 \mathrm{~N}$ solution of hydrogen chloride in purified chloroform, in agreement with the properties of other orthoesters [19], it was converted into the acetylglycosyl chloride. Four minutes after dissolution the specific rotation of the compound had changed from $+25.3^{\circ}$ to $+62.7^{\circ}$. The solution, upon evaporation, yielded crystalline heptaacetyl- $\alpha$ neolactosyl chloride, which was purified by recrystallization from ethyl acetate and identified by melting point and measurements of optical rotation.

Hydrolyses by acid and by alkali were carried out in approximately the same manner as that described for tetracetyl- $d$ - $\alpha$-guloheptose methyl orthoacetate. In the analysis of the crystalline compound, $0.2-\mathrm{g}$ samples were dissolved in 10-ml quantities of methyl alcohol in glass-stoppered flasks and the hydrolyses were carried out on these solutions. By alkaline hydrolysis $0.2 \mathrm{~g}$ of hexaacetylneolactose methyl orthoacetate required $18.61 \mathrm{ml}$ of $0.1 \mathrm{~N}$ alkali, and by acid hydrolysis $0.2 \mathrm{~g}$ of the compound was equivalent to $21.39 \mathrm{ml}$ of $0.1 \mathrm{~N}$ alkali. Calculated for six acetyl groups, $18.45 \mathrm{ml}$ of $0.1 \mathrm{~N}$ alkali; for seven acetyl groups, $21.52 \mathrm{ml}$ of $0.1 \mathrm{~N}$ alkali.

\section{METHYL HEPTAACETYL- $\beta$-NEOLACTOPYRANOSIDE}

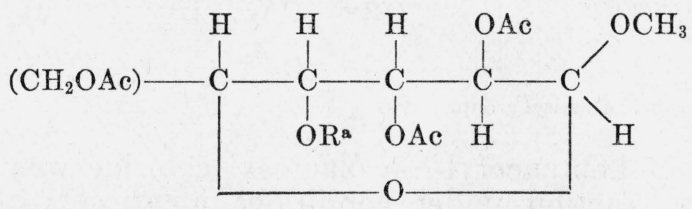

a " $R$ " is the tetraacetyl- $\beta-d$-galactosyl group.

The second crystalline substance separated from the product of the Koenigs-Knorr reaction on heptaacetyl- $\alpha$-neolactosyl chloride, melting point $168^{\circ}$ to $174^{\circ} \mathrm{C}$, was recrystallized several times from chloroform with the addition of ether, and finally from a large volume of methyl alcohol. When pure, methyl heptaacetyl- $\beta$-neolactoside forms rectangular prisms (fig. $2, E$ ) which melt at $179^{\circ} \mathrm{C}$ and give $[\alpha]_{D}^{20}=-14.5^{\circ}$. Unlike the orthoacetate, this compound did not react rapidly with hydrogen chloride. When it was treated with a chloroform solution of hydrogen chloride, there was no change in rotation, and the solution, after evaporation, yielded crystals identical with those of the original compound. Analysis: Calculated for $\mathrm{C}_{27} \mathrm{H}_{38} \mathrm{O}_{18}$ : C, 49.84; H, 5.89. Found: C, 49.70; H, 5.91.

Analysis by either acid or alkaline hydrolysis indicated seven acetyl groups. In the acid hydrolysis, a 0.2 -g sample was equivalent to 21.64 $\mathrm{ml}$ of $0.1 \mathrm{~N}$ alkali; in the alkaline hydrolysis, to $21.66 \mathrm{ml}$ of alkali. Calculated for seven acetyl groups, $21.52 \mathrm{ml}$ of $0.1 \mathrm{~N}$ alkali.

Inasmuch as the hydroxyl on carbon 4 of the altrose unit of neolactose is blocked by a galactosyl group, the compound cannot be a furanoside and must be a pyranoside. The specific rotation of the beta modification of methyl heptaacetylneolactopyranoside may be calculated from the molecular rotations of pentaacetyl- $\alpha-d$-altrose $(+24,600)$ [27], octaacetyl- $\alpha$-neolactose $(+36,200)$ [23], and methyl tetraacetyl- $\beta-d$-altropyranoside $(-22,100)$ [27]. According to the 


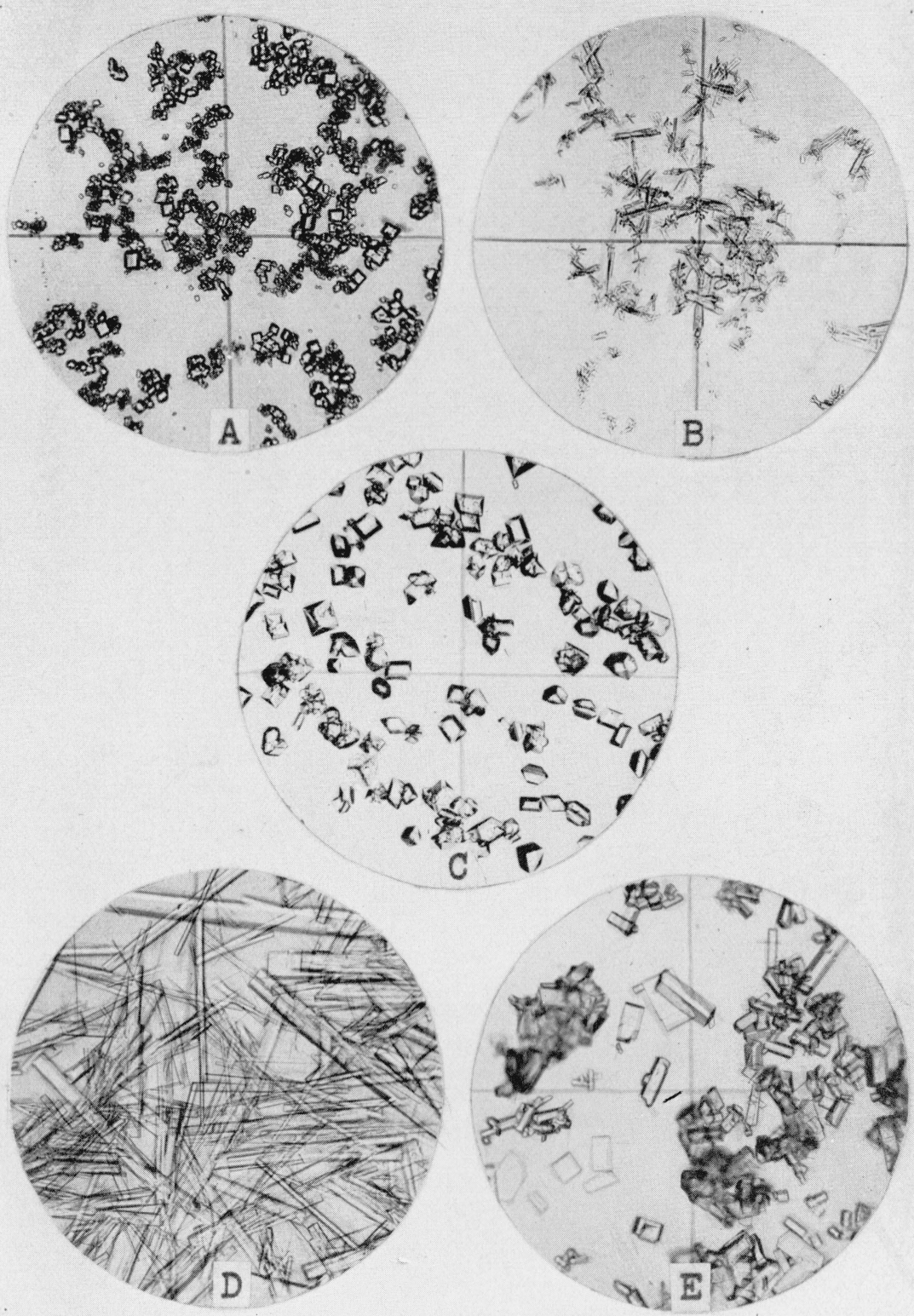

Figure 2.-Photomicrographs of new compounds.

$A$, Hexaacetyl- $\alpha-d-\alpha$-guloheptopyranose.

$B$, Pentaacetyl- $\alpha$ - $d-\alpha$-guloheptopyranosyl bromide.

$C$, Totraacetyl- $d$ - $\alpha$-guloheptose methyl 1,2-orthoacetate.

$D$, Hexaacetylneolactose methyl 1,2-orthoacetate.

$E$, Methyl heptaacetyl- $\beta$-neolactopyranoside. 
principle of optical superposition as applied to the sugars by Hudson, the difference in the molecular rotations of pentaacetyl- $\alpha$ - $d$-altrose $(+24,600)$ and octaacetyl- $\alpha$-neolactose $(+36,200)$ should equal approximately the difference in the molecular rotations of methyl tetraacetyl- $\beta$ - $d$-altropyranoside $(-22,100)$ and methyl heptaacetyl- $\beta$ neolactopyranoside. Using the molecular rotations for the first three compounds, the molecular rotation of the fourth is calculated to be $-10,500$. This value corresponds to a specific rotation of $-16.1^{\circ}$, which compares favorably with our experimental value of $-14.5^{\circ}$, and therefore the compound is properly designated methyl heptaacetyl$\beta$-neolactopyranoside.

\section{SUMMARY}

$\alpha-d-\alpha$-Guloheptose was acetylated by the low-temperature pyridine method to yield crystalline hexaacetyl- $\alpha-d$ - $\alpha$-guloheptopyranose, melting point $126^{\circ} \mathrm{C},[\alpha]_{D}^{20}=-62.8^{\circ}$. The new compound, when treated with hydrogen bromide in acetic acid, gave crystalline pentaacetyl- $\alpha$ - $d$ - $\alpha$-guloheptopyranosyl bromide, melting point $139^{\circ}$ to $140^{\circ} \mathrm{C},[\alpha]_{D}^{20}=-124^{\circ}$.

The mechanism of orthoester formation was discussed in light of the opposite-face concept for the Walden inversion, and a test of the mechanism was made by application of the Koenigs-Knorr reaction to the new pentaacetyl- $\alpha-d-\alpha$-guloheptopyranosyl bromide and to heptaacetyl- $\alpha$-neolactosyl chloride. Both of these compounds have configurations which allow the acetyl group of carbon 2 to approach the face of carbon 1 opposite the replaceable halogen, and hence should yield orthoacetates according to the opposite-face hypothesis for the formation of orthoesters. On treatment with methyl alcohol in the presence of silver carbonate, pentaacetyl- $\alpha-d$ - $\alpha$-guloheptopyranosyl bromide gave an almost quantitative yield of tetraacetyl$d$ - $\alpha$-guloheptose methyl 1,2 -orthoacetate, melting point $106^{\circ} \mathrm{C}$, $[\alpha]_{D}^{20}=+3.2^{\circ}$. Heptaacetyl- $\alpha$-neolactosyl chloride under like treatment gave about 70 percent of hexaacetylneolactose methyl 1,2-orthoacetate, melting point $121^{\circ}$ to $122^{\circ} \mathrm{C},[\alpha]_{D}^{20}=+25.3^{\circ}$, and about 30 percent of methyl heptaacetyl- $\beta$-neolactopyranoside, melting point $179^{\circ} \mathrm{C},[\alpha]_{D}^{20}=-14.5^{\circ}$. Presumably these new compounds are formed, respectively, by an intramolecular orthoester reaction and by a competitive extramolecular glycosidic reaction. Since neolactose is a substituted altrose, and orthoesters of the altrose configuration have not heretofore been prepared, the formation of the orthoacetate is convincing evidence for the validity of the opposite-face mechanism, as previously postulated by one of the authors to explain orthoester formation.

The new orthoacetates show the reactions characteristic of the sugar methyl orthoacetates, including stability to alkaline hydrolysis and the formation of the normal glycosyl halide on treatment with hydrogen chloride. Tetraacetyl- $d$ - $\alpha$-guloheptose methyl orthoacetate, on treatment with hydrogen chloride, gave a crystalline material, presumably pentaacetyl- $\alpha-d$ - $\alpha$-guloheptosyl chloride, which is being investigated further; and hexaacetylneolactose methyl orthoacetate yielded heptaacetyl- $\alpha$-neolactosyl chloride. 


\section{REFERENCES}

[1] E. Fischer and K. Delbrück, Ber. deut. chem. Ges. 42, 2776 (1909).

[2] W. N. Haworth, E. L. Hirst, and M. Stacey, J. Chem. Soc. 1931, 2864.

[3] P. Walden, Ber. deut. chem. Ges. 29, 133 (1896).

[4] P. Walden, J. chim. phys. 9, 160 (1911).

[5] P. F. Frankland, J. Chem. Soc. 103, 713 (1913).

[6] A. Werner, Ber. deut. chem. Ges. 44, 881 (1911).

[7] W. E. Garner, Proc. Chem. Soc. 29, 198 (1913).

[8] L. P. Hammett, Physical Organic Chemistry (McGraw-Hill Book Co., New York, N.'Y., 1940).

[9] H. S. Isbell, Ann. Rev. Biochem. 9, 65 (1940).

[10] W. Koenigs and E. Knorr, Ber. deut. chem. Ges. 34, 957, (1901).

[11] E. Fischer and E. F. Armstrong, Ber. deut. chem. Ges. 34, 2885 (1901).

[12] E. Fischer, M. Bergmann, and A. Rabe, Ber. deut. chem. Ges. 53, 2362 (1920).

[13] K. Freudenberg and E. Braun, Naturwissenschaften 18, 393 (1930).

[14] W. N. Haworth, E. L. Hirst, and E. J. Miller, J. Chem. Soc. 1929, 2469.

[15] H. G. Bott, W. N. Haworth, and E. L. Hirst, J. Chem. Soc. 1930, 1395.

[16] K. Freudenberg and H. Scholz, Ber. deut. chem. Ges. 63, 1969 (1930).

[17] P. A. Levene and M. L. Wolfrom, J. Biol. Chem. 78, 525 (1928).

[18] J. K. Dale, J. Am. Chem. Soc. 46, 1046 (1924).

[19] H. S. Isbell, BS J. Research 7, 1115 (1931) RP392.

[20] P. A. Levene and R. S. Tipson, J. Biol. Chem. 92, 109 (1931).

[21] W. W. Pigman and H. S. Isbell, J. Research NBS 19, 189 (1937) RP1021.

[22] F. Micheel and H. Micheel, Ber. deut. chem. Ges. 63, 386 (1930); 65, 253 (1932).

[23] A. Kunz and C. S. Hudson, J. Am. Chem. Soc. 48, 1978, 2435 (1926).

[24] N. K. Richtmyer and C. S. Hudson, J. Am. Chem. Soc. 5\%, 1716 (1935).

[25] H. S. Isbell, J. Chem. Education 12, 96 (1935).

[26] H. S. Isbell, J. Research NBS 19, 639 (1937) RP1052.

[27] N. K. Richtmyer and C. S. Hudson, J. Am. Chem. Soc. 63, 1727 (1941).

[28] C. S. Hudson, J. Am. Chem. Soc. 48, 1434 (1926).

[29] B. Helferich, E. Bohn, and S. Winkler, Ber. deut. chem. Ges. 63, 990 (1930).

[30] D. D. Reynolds and W. L. Evans, J. Am. Chem. Soc. 60, 2559 (1938).

[31] H. B. MacPhillamy and R. C. Elderfield, J. Org. Chem. 4, 150 (1939).

Washington, September 2, 1941. 